\title{
More of Dedekind: His Series Test in Normed Spaces
}

\author{
Robert Kantrowitz ${ }^{1}$ and Michael M. Neumann ${ }^{2}$ \\ ${ }^{1}$ Department of Mathematics, Hamilton College, 198 College Hill Road, Clinton, NY 13323, USA \\ ${ }^{2}$ Department of Mathematics and Statistics, Mississippi State University, Mississippi State, MS 39762, USA
}

Correspondence should be addressed to Michael M. Neumann; neumann@math.msstate.edu

Received 6 December 2015; Accepted 5 May 2016

Academic Editor: Attila Gilányi

Copyright (C) 2016 R. Kantrowitz and M. M. Neumann. This is an open access article distributed under the Creative Commons Attribution License, which permits unrestricted use, distribution, and reproduction in any medium, provided the original work is properly cited.

Dedekind's test for infinite series has a canonical interpretation in the context of normed spaces. It is shown that his test holds in a normed space precisely when the space is complete.

\section{Introduction}

The theoretical underpinning of all of calculus is the completeness property of the field of real numbers. Recent work has probed deeply into the many ways in which completeness and the attendant Archimedean property are manifested in $\mathbb{R}$. The real analysis in reverse program, as described and promoted in [1], has brought to the fore a wealth of results dealing with Dedekind completeness, Cauchy completeness, and the Archimedean property in the context of arbitrary ordered fields; see also [2-5].

As recent contributions to this program, it was shown in [6] that an ordered field $\mathbb{F}$ has the Archimedean property precisely when the geometric series test holds in $\mathbb{F}$ and in an article under review by the authors that $\mathbb{F}$ is Dedekind complete if and only if both the geometric series test and Dedekind's test for infinite series hold in $\mathbb{F}$. It was also observed in that paper that there are many non-Archimedean ordered fields for which Dedekind's test is valid for the simple reason that every convergent sequence in such a field is eventually constant.

In the present paper, we discuss the fate of these two convergence tests in the setting of normed spaces and normed algebras, now, of course, with a shift of emphasis away from Dedekind completeness to completeness in the sense of Cauchy, also known as sequential completeness.

\section{Main Results}

The geometric series test has an obvious formulation in the context of normed algebras and lies at the heart of spectral theory. In fact, by Proposition 3.3 of [7], the validity of this test characterizes the class of spectral algebras, an important class of normed algebras that is strictly larger than that of Banach algebras. As further detailed in the monograph [8] by Palmer, there are quite a few interesting examples of incomplete normed algebras for which the geometric series test holds.

Here we focus on the role of Dedekind's test for infinite series in normed spaces and normed algebras. Motivated by the scalar version of this result $[9,10]$, we say that Dedekind's test holds in a normed space $X$ over the scalar field $\mathbb{F}=\mathbb{R}$ or $\mathbb{C}$ if the series

$$
\sum_{n=1}^{\infty} a_{n} x_{n}
$$

converges in $X$ whenever $\left(a_{n}\right)_{n \in \mathbb{N}}$ is a sequence in $\mathbb{F}$ for which the series $\sum_{n=1}^{\infty} a_{n}$ converges and $\left(x_{n}\right)_{n \in \mathbb{N}}$ is a sequence of bounded variation in $X$, in the sense that

$$
\sum_{n=1}^{\infty}\left\|x_{n+1}-x_{n}\right\|<\infty
$$

An adaptation of the standard scalar argument will show that Dedekind's test holds if $X$ is complete. In fact, first observe that the sequence of partial sums $\left(A_{n}\right)_{n \in \mathbb{N}}$ of the scalar series $\sum_{n=1}^{\infty} a_{n}$ is convergent and thus bounded by some constant 
$M>0$. Moreover, since Abel's classical partial summation formula [9] extends to the present context, we have

$$
\sum_{n=1}^{m} a_{n} x_{n}=A_{m} x_{m+1}-\sum_{n=1}^{m} A_{n}\left(x_{n+1}-x_{n}\right)
$$

for all $m \in \mathbb{N}$. Hence it remains to see that the two sequences on the right hand side converge as $m \rightarrow \infty$. But this is indeed the case, since all sequences of bounded variation in $X$ are Cauchy and hence convergent in the Banach space $X$, while the estimate

$$
\sum_{n=1}^{\infty}\left|A_{n}\right|\left\|x_{n+1}-x_{n}\right\| \leq M \sum_{n=1}^{\infty}\left\|x_{n+1}-x_{n}\right\|<\infty
$$

guarantees that the sequence of partial sums of the series $\sum_{n=1}^{\infty} A_{n}\left(x_{n+1}-x_{n}\right)$ is Cauchy and hence convergent in $X$. We now turn our attention to the converse of the result just established.

Theorem 1. A normed space $X$ is complete precisely when Dedekind's series test holds for $X$.

The following lemma clears away the technicalities of what remains to be seen. Since the proof of the corresponding result for the case of ordered fields relies heavily on the order structure, a new argument is needed here.

To offer some idea of the intuition behind the somewhat technical condition governing Lemma 2 below, we follow a referee's suggestion and say that a sequence $\left(u_{n}\right)_{n \in \mathbb{N}}$ in a normed space $X$ is wandering if it does not converge to any of its terms and restless if, for all real numbers $c>1$ and integers $r \in \mathbb{N}$, there exists some $s \in \mathbb{N}$ with $s>r$ such that the estimate

$$
\begin{aligned}
& c^{2}\left\|c\left(u_{p}-u_{q}\right)-\left(u_{q}-u_{s}\right)\right\| \\
& \leq\left\|c\left(u_{q}-u_{s}\right)-\left(u_{s}-u_{r}\right)\right\|
\end{aligned}
$$

holds for all $p, q \in \mathbb{N}$ with $p, q \geq s$. Lemma 2 then confirms that all wandering Cauchy sequences are restless, while every restless sequence is either wandering or eventually constant.

Lemma 2. Let $\left(u_{n}\right)_{n \in \mathbb{N}}$ be a Cauchy sequence in a normed space $X$, suppose that $c$ is a real number with $c>1$, and let $r \in \mathbb{N}$ be a given integer. If $\left(u_{n}\right)_{n \in \mathbb{N}}$ does not converge to $u_{r}$, then there exists some $s \in \mathbb{N}$ with $s>r$ such that the inequality $(\diamond)$ is satisfied for all $p, q \in \mathbb{N}$ with $p, q \geq s$. The converse holds provided that the terms of the sequence $\left(u_{n}\right)_{n \in \mathbb{N}}$ are not eventually $u_{r}$.

Proof. First suppose that $u_{r}$ is not the limit of the sequence $\left(u_{n}\right)_{n \in \mathbb{N}}$. Then there exists some $\varepsilon>0$ such that, for each $m \in$ $\mathbb{N}$, we can find some $k \in \mathbb{N}$ for which $k>m$ and $\left\|u_{k}-u_{r}\right\| \geq \varepsilon$. On the other hand, since $\left(u_{n}\right)_{n \in \mathbb{N}}$ is a Cauchy sequence, there exists some $\ell \in \mathbb{N}$ such that

$$
\left\|u_{p}-u_{q}\right\| \leq \frac{\varepsilon}{c^{3}+c^{2}+c}
$$

for all $p, q \in \mathbb{N}$ with $p, q \geq \ell$. With the choice $m=\max \{\ell, r\}$ we finally find some $s \in \mathbb{N}$ for which $s>m$ and $\left\|u_{s}-u_{r}\right\| \geq \varepsilon$.
We claim that this $s$ has the desired properties. Clearly, $s>r$. Moreover, for all $p, q \in \mathbb{N}$ with $p, q \geq s$, we have $p, q \geq \ell$ and therefore

$$
c^{2}\left\|c\left(u_{p}-u_{q}\right)-\left(u_{q}-u_{s}\right)\right\| \leq \frac{c^{3}+c^{2}}{c^{3}+c^{2}+c} \varepsilon .
$$

On the other hand, we obtain

$$
\begin{aligned}
& \left\|c\left(u_{q}-u_{s}\right)-\left(u_{s}-u_{r}\right)\right\| \geq\left\|u_{s}-u_{r}\right\|-\left\|c\left(u_{q}-u_{s}\right)\right\| \\
& \quad \geq \varepsilon-\frac{c \varepsilon}{c^{3}+c^{2}+c}=\frac{c^{3}+c^{2}}{c^{3}+c^{2}+c} \varepsilon
\end{aligned}
$$

and thus the desired inequality $(\diamond)$.

To establish the final claim, suppose that $\left(u_{n}\right)_{n \in \mathbb{N}}$ converges to $u_{r}$ and that there exists some $s \in \mathbb{N}$ with $s>r$ such that the estimate $(\diamond)$ holds for all $p, q \in \mathbb{N}$ with $p, q \geq s$. Our task is to show that the terms of the sequence $\left(u_{n}\right)_{n \in \mathbb{N}}$ are eventually $u_{r}$. Now, passing to the limit as $q \rightarrow \infty$ in $(\diamond)$ yields

$$
c^{2}\left\|c\left(u_{p}-u_{r}\right)-\left(u_{r}-u_{s}\right)\right\| \leq\left\|c\left(u_{r}-u_{s}\right)-\left(u_{s}-u_{r}\right)\right\|
$$

for all $p \geq s$. In particular, the choice $p=s$ then shows that $u_{s}=u_{r}$. With this substitution, another look at the preceding estimate reveals that actually $u_{p}=u_{r}$ for all $p \geq s$. This completes the argument.

Proof of Theorem 1. We already established the validity of Dedekind's test in arbitrary Banach spaces. For the converse, suppose that Dedekind's test holds for the normed space $X$, and consider an arbitrary Cauchy sequence $\left(u_{n}\right)_{n \in \mathbb{N}}$ in $X$. To see that this sequence converges in $X$, we may certainly assume that it is wandering. This allows us to employ Lemma 2 to construct a certain subsequence by induction. We start by choosing any $c>1$ and letting $n(1)=1$. For the inductive step, if the integer $n(k)$ for some $k \in \mathbb{N}$ has already been chosen, then we apply Lemma 2 to $r=n(k)$ to obtain an integer $s=n(k+1)$ with $n(k+1)>n(k)$ such that the a priori estimate

$$
\begin{aligned}
& c^{2}\left\|c\left(u_{p}-u_{q}\right)-\left(u_{q}-u_{n(k+1)}\right)\right\| \\
& \quad \leq\left\|c\left(u_{q}-u_{n(k+1)}\right)-\left(u_{n(k+1)}-u_{n(k)}\right)\right\|
\end{aligned}
$$

holds for all $p, q \in \mathbb{N}$ with $p, q \geq n(k+1)$. Once the sequence of integers $n(k)$ has been found in this manner, for each $k \in \mathbb{N}$ we may invoke the preceding estimate with the choice $p=$ $n(k+3)$ and $q=n(k+2)$ to obtain

$$
\begin{aligned}
& c^{2}\left\|c\left(u_{n(k+3)}-u_{n(k+2)}\right)-\left(u_{n(k+2)}-u_{n(k+1)}\right)\right\| \\
& \leq\left\|c\left(u_{n(k+2)}-u_{n(k+1)}\right)-\left(u_{n(k+1)}-u_{n(k)}\right)\right\| .
\end{aligned}
$$

Thus, with the definition

$$
x_{k}=c^{k}\left(u_{n(k+1)}-u_{n(k)}\right) \in X
$$

for all $k \in \mathbb{N}$, the last estimate may be rewritten in the form

$$
\left\|x_{k+2}-x_{k+1}\right\| \leq \frac{1}{c}\left\|x_{k+1}-x_{k}\right\|
$$


for all $k \in \mathbb{N}$. But this ensures that

$$
\left\|x_{k+2}-x_{k+1}\right\| \leq \frac{1}{c^{k}}\left\|x_{2}-x_{1}\right\|
$$

for all $k \in \mathbb{N}$ and thus shows that the sequence $\left(x_{n}\right)_{n \in \mathbb{N}}$ is of bounded variation in $X$. On the other hand, the geometric series

$$
\sum_{k=1}^{\infty} c^{-k}
$$

converges in $\mathbb{F}$, because $c>1$. Since we suppose that Dedekind's test holds for $X$, we conclude that the sequence of partial sums

$$
\sum_{k=1}^{m} c^{-k} x_{k}=\sum_{k=1}^{m}\left(u_{n(k+1)}-u_{n(k)}\right)=u_{n(m+1)}-u_{1}
$$

converges in $X$ as $m \rightarrow \infty$. But this means that the Cauchy sequence $\left(u_{n}\right)_{n \in \mathbb{N}}$ has a convergent subsequence and thus itself converges to the same limit. This establishes the completeness of $X$.

We finally discuss a natural variant of Dedekind's test for the case of a normed algebra $A$ with identity. Let us say that the algebra version of Dedekind's test holds in $A$ provided that the series

$$
\sum_{n=1}^{\infty} a_{n} b_{n}
$$

is convergent whenever $\left(a_{n}\right)_{n \in \mathbb{N}}$ and $\left(b_{n}\right)_{n \in \mathbb{N}}$ are both sequences in $A$ such that $\sum_{n=1}^{\infty} a_{n}$ converges in $A$ and $\left(b_{n}\right)_{n \in \mathbb{N}}$ is of bounded variation. We obtain the following counterpart of Theorem 1 .

Corollary 3. A normed algebra $A$ with identity is complete precisely when the algebra version of Dedekind's test holds for A.

Proof. First, an inspection of our previous argument for Dedekind's test in normed spaces reveals that the algebra version of this test holds in any Banach algebra. Conversely, suppose that $A$ is a normed algebra over $\mathbb{F}=\mathbb{R}$ or $\mathbb{C}$ with identity element $e$ such that the algebra version of Dedekind's test is valid. Clearly, for every sequence $\left(a_{n}\right)_{n \in \mathbb{N}}$ in $\mathbb{F}$ for which $\sum_{n=1}^{\infty} a_{n}$ converges in $\mathbb{F}$, the series $\sum_{n=1}^{\infty} a_{n} e$ converges in $A$. Thus the former vector space version of Dedekind's test holds in $A$, which implies, by Theorem 1 , that $A$ is complete.

It is not difficult to see that the preceding result remains true if, in the definition of the algebra version of Dedekind's test, the roles of the two sequences are interchanged. We leave the proof to the interested reader. As a consequence of Corollary 3, we obtain that, for any incomplete spectral algebra with identity, the algebra version of Dedekind's test fails to hold in general, while we know from Proposition 3.3 of [7] that the geometric series test is valid for such an algebra. We conclude with a specific example to illustrate this phenomenon.
Example 4. Given the compact disc $\Delta=\{z \in \mathbb{C}:|z| \leq$ $1 / 2\}$, let $A$ consist of all rational complex-valued functions on $\Delta$ with poles outside $\Delta$. Evidently, $A$ becomes a normed algebra with identity when endowed with pointwise algebra operations and the supremum norm $\|\cdot\|_{\infty}$. For every $u \in A$ with $\|u\|_{\infty}<1$, the series $\sum_{n=0}^{\infty} u^{n}$ converges uniformly on $\Delta$ to the rational function $1 /(1-u) \in A$. This shows that the geometric series test holds in $A$ and hence that $A$ is spectral. On the other hand, let $a_{n}(z)=z^{n}$ and $b_{n}(z)=1 / n$ ! for all $z \in \Delta$ and $n \in \mathbb{N}_{0}$. Then clearly $a_{n}$ and $b_{n}$ belong to $A$, the series $\sum_{n=0}^{\infty} a_{n}$ converges uniformly on $\Delta$ to the function $a \in A$ given by $a(z)=1 /(1-z)$ for all $z \in \Delta$, and $\left(b_{n}\right)_{n \in \mathbb{N}_{0}}$ is certainly of bounded variation in $A$. But the series $\sum_{n=0}^{\infty} a_{n} b_{n}$ does not converge in $A$, since it converges uniformly on $\Delta$ to the exponential function. Consequently, the algebra version of Dedekind's test is invalid in $A$, as predicted by Corollary 3 .

\section{Competing Interests}

The authors declare that they have no competing interests.

\section{References}

[1] J. Propp, "Real analysis in reverse," American Mathematical Monthly, vol. 120, no. 5, pp. 392-408, 2013.

[2] P. L. Clark and N. J. Diepeveen, "Absolute convergence in ordered fields," American Mathematical Monthly, vol.121, no. 10, pp. 909-916, 2014.

[3] M. Deveau and H. Teismann, " $72+42$ : characterizations of the completeness and Archimedean properties of ordered fields," Real Analysis Exchange, vol. 39, no. 2, pp. 261-304, 2013-2014.

[4] O. Riemenschneider, "37 elementare axiomatische Charakterisierungen des reellen Zahlkörpers," Mitteilungen der Mathematischen Gesellschaft in Hamburg, vol. 20, pp. 71-95, 2001.

[5] H. Teismann, "Toward a more complete list of completeness axioms," The American Mathematical Monthly, vol. 120, no. 2, pp. 99-114, 2013.

[6] R. Kantrowitz and M. M. Neumann, "Another face of the Archimedean property," The College Mathematics Journal, vol. 46, no. 2, pp. 139-141, 2015.

[7] T. W. Palmer, "Spectral algebras," The Rocky Mountain Journal of Mathematics, vol. 22, no. 1, pp. 293-328, 1992.

[8] T. W. Palmer, Banach Algebras and the General Theory of * Algebras, Volume I: Algebras and Banach Algebras, Cambridge University Press, Cambridge, UK, 1994.

[9] T. M. Apostol, Mathematical Analysis, Addison-Wesley, Reading, Mass, USA, 2nd edition, 1974.

[10] K. Knopp, Infinite Sequences and Series, Dover Publications Inc, New York, NY, USA, 1956. 


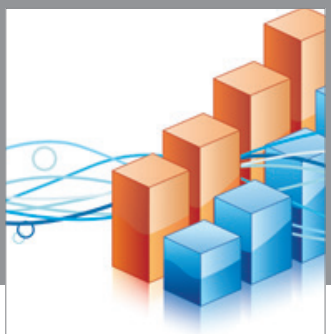

Advances in

Operations Research

vatem alat4

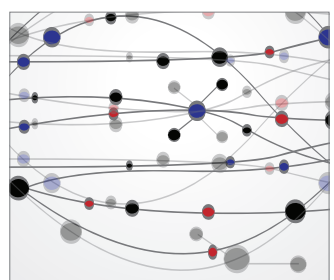

\section{The Scientific} World Journal
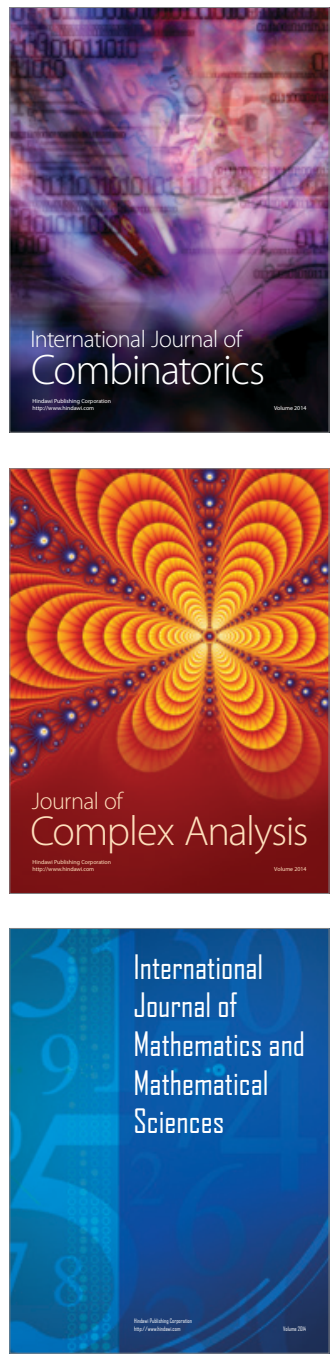
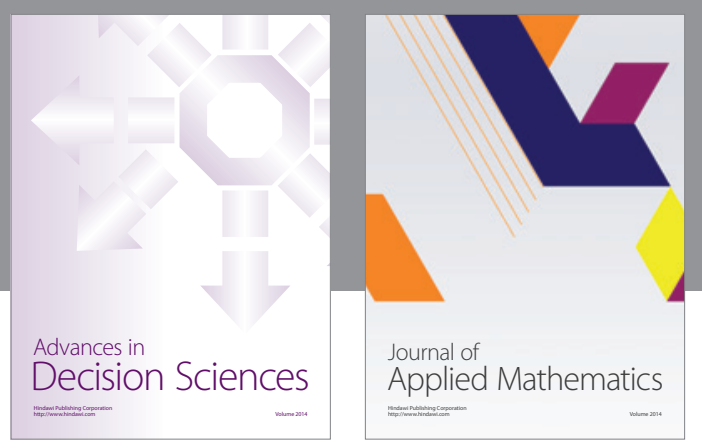

Algebra

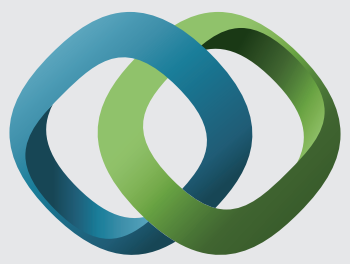

\section{Hindawi}

Submit your manuscripts at

http://www.hindawi.com
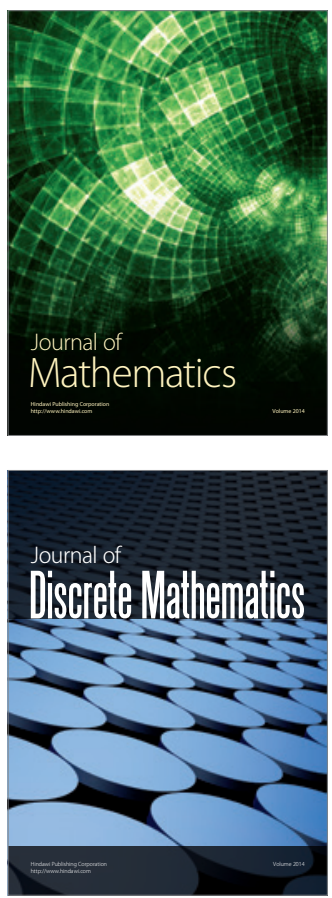

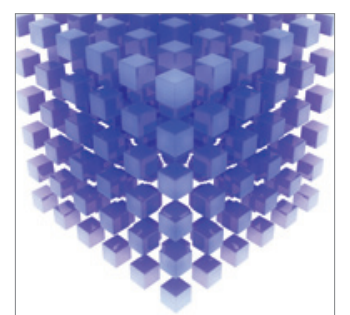

Mathematical Problems in Engineering
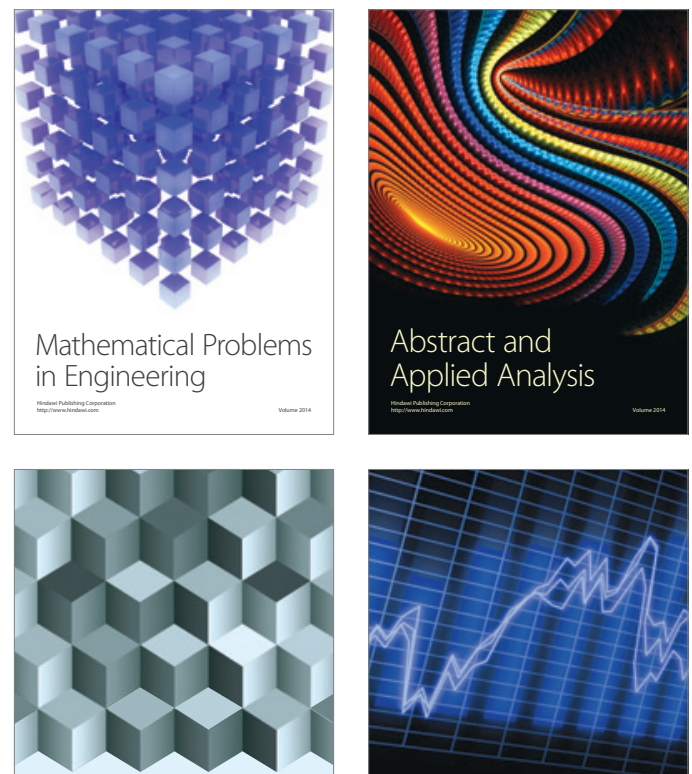

Journal of

Function Spaces

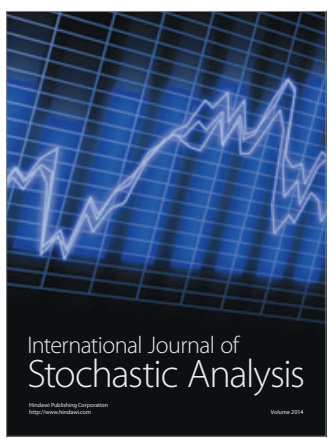

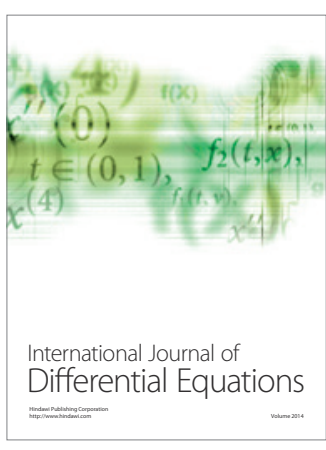
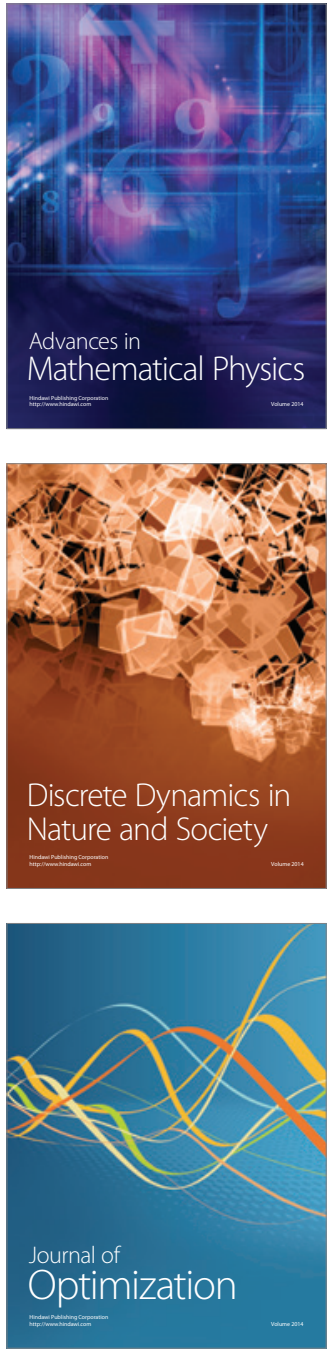